\title{
Prevalence of parenthood in wheelchair-dependent persons with long-term spinal cord injury in the Netherlands
}

\author{
K. van den Borne ${ }^{1}$ - I. Brands ${ }^{1}$ D. Spijkerman ${ }^{2}$ J. J. E. Adriaansen ${ }^{3}$ K. Postma ${ }^{2,4}$ - H. J. G. van den Berg-Emons ${ }^{1,4}$
}

Received: 16 September 2017 / Revised: 20 December 2017 / Accepted: 23 December 2017 / Published online: 19 January 2018

(c) International Spinal Cord Society 2018

\begin{abstract}
Study design Multicenter cross-sectional study.

Objectives To determine the prevalence of parenthood in long-term wheelchair-dependent persons who sustained a spinal cord injury (SCI) during their reproductive years. Secondary aims were to (1) explore patient-specific and disease-related factors associated with parenthood after SCI; and (2) quantify fertility aids used by men with SCI.

Setting Eight specialized SCI rehabilitation centers in the Netherlands.

Methods Questionnaires and physical examination were applied in 255 persons with SCI. Prevalence rates of parenthood among the general Dutch population were used for comparison. Logistic regression analyses were used to explore factors associated with parenthood after SCI.

Results Prevalence of parenthood in SCI was 50\% in men and $45 \%$ in women, which was significantly $(P<0.05)$ lower than rates in the general population (74\% in men and $81 \%$ in women). Among the parents with SCI, most (66\% of males and $72 \%$ of females) of them had children after SCI. Parenting children after SCI was associated with partnership (OR $=14.5, P$ $<.001$ [men]; $\mathrm{OR}=3.7, P=.05$ [women]), normal micturition ( $\mathrm{OR}=4.9, P=.02$ [men]), incomplete lesion $(\mathrm{OR}=5.4, P$ $=.03$ [women]), and paraplegia $(\mathrm{OR}=7.3, P=.02$ [women]). The most frequently used methods for ejaculation and fertilization were electroejaculation (29\%) and intracytoplasmatic sperm injection (23\%).

Conclusions Prevalence of parenthood in SCI persons is low. However, half of the persons with SCI do become parents, with most doing so following SCI. Demographic and disease-related factors may contribute to this.
\end{abstract}

\section{Introduction}

The annual incidence of spinal cord injury (SCI) in the Netherlands is approximately 400 cases, of which 185 are traumatic [1]. Of those affected by traumatic SCI, most of them are men in their prime reproductive years. SCI leaves most of these men with impaired fertility, with only a minority being able to have children through sexual intercourse $[2,3]$. In women with SCI, a 3-5-month period of

I. Brands

I.brands@libranet.nl

Libra Rehabilitation \& Audiology, Eindhoven, The Netherlands

2 Rijndam Rehabilitation, Rotterdam, The Netherlands

3 Brain Center Rudolf Magnus and Center of Excellence in Rehabilitation Medicine, De Hoogstraat Rehabilitation, Utrecht, The Netherlands

4 Erasmus Medical Center, Rotterdam, The Netherlands amenorrhea is followed by resumption of fertility comparable to that of unaffected women $[4,5]$.

Fertility problems in men are primarily due to erectile dysfunction, ejaculatory dysfunction, or poor semen quality $[6,7]$. The capacity for reflexogenic, psychogenic, and nocturnal erections is decreased and depends upon the level of neurologic damage and injury completeness [6, 7]. Although the causes of poor semen quality (low volume and impaired sperm motility) are not well understood, urinary tract infections, testicular hyperthermia, sex hormone changes, sperm antibodies, sperm stagnation, and stress may play a role [7]. Because of decreased fertility, assisted reproductive technology is often needed for men with SCI to father children [4]. Penile vibration stimulation (PVS) is typically the first recommended treatment, followed by electroejaculation (EE) [4]. If these techniques fail, assisted reproductive techniques such as intrauterine insemination (IUI), in vitro fertilization (IVF), testicular sperm aspiration/ extraction (TESA/TESE), or intracytoplasmatic sperm injection (ICSI) can be used [7]. Effectiveness studies of 
assisted reproductive technology in men affected by SCI show partner pregnancy rates of 49-60\% and live birth rates of $40-63 \%$ [4, 6, 8]. PVS with multiple ovulation cycles is associated with pregnancy rates of $25-61 \%$ and ICSI with rates of 20-40\% [7, 9-12]. In contrast, PVS or EE with IUI resulted in a live birth rate of less than $10 \%$ [7, 13].

For both men and women, disabilities associated with SCI may pose other barriers to parenthood, including concerns about caring for a child, social burden [14, 15], and discouragement from healthcare professionals $[16,17]$. Furthermore, we hypothesized that physical challenges common among those with SCI (e.g., bowel and bladder problems) may influence intimacy and the ability for sexual relationships, which in turn may restrict wishes and opportunities for parenthood. However, no studies have addressed the effect of those aspects on parenthood among those with SCI.

There are little data available about the prevalence of parenthood in men and women with SCI who sustained injuries during their reproductive years, irrespective of their use of assisted reproductive technology. Nikas et al. found a 47\% prevalence of fatherhood among World War II veterans with paraplegia and tetraplegia [18], at which time assisted reproductive techniques were limited. In their study of 253 men and women with SCI, Pebdani et al. found a parenthood prevalence of $53 \%$ for men and $60 \%$ for women [17]. They found that only $30 \%$ of participants had children after SCI, despite only half of them having achieved their parenthood goals prior to SCI [17].

The aim of this study was to estimate the prevalence of parenthood in a cohort of men and women with long-term SCI (greater than 10 years post injury), who sustained their injury between the ages of 18 and 35 years. The secondary aims were to 1) explore personal and disease-related factors associated with parenthood prevalence; and 2) quantify fertility aids used by men with SCI who fathered children. We focused on men and women who were wheelchair dependent, as this comprises the largest group of patients with SCI [19]. We hypothesized that parenthood prevalence among wheelchair-dependent men and women with SCI, but particularly men, would be considerably lower than that of able-bodied persons because of fertility problems and other barriers. Knowledge of parenthood prevalence and associated factors may improve clinical counseling for patients with SCI who wish to have children.

\section{Methods}

\section{Design and participants}

This cross-sectional study was part of the Active LifestyLe Rehabilitation Interventions in an aging Spinal Cord Injury (ALLRISC) project, which describes secondary health conditions and care needs of men and women with longterm SCI (greater than 10 years post injury) in the Netherlands [20]. For this project, data were collected from 282 patients from 2011 to 2013 in the eight Dutch rehabilitation centers with specialized SCI units. Inclusion criteria were 1) the presence of SCI; 2) age at injury of 18-35 years; 3) greater than 10 years since SCI; 4) current age of 28-65 years; and 5) wheelchair dependence for distances greater than $500 \mathrm{~m}$ [19]. Persons were excluded if they had insufficient mastery of the Dutch language that precluded understanding of test instructions. The current study focused on parenthood prevalence.

\section{Procedure}

Participants were invited for a 1-day visit to the rehabilitation center for evaluation by a rehabilitation physician for a long-term SCI checkup that included neurological examination based on the International Standards for Neurological Classification of SCI (ISNCSCI) and oral interview [21]. Participants were asked to complete several questionnaires before the appointment. These questionnaires, which were developed for the purpose of this study, are available upon request [20]. The study protocol was approved by the Medical Ethics Committee of the University Medical Centre Utrecht and participants provided written informed consent [20].

\section{Outcome measures}

\section{Parenthood prevalence}

A yes/no questionnaire was used to measure whether participants had children and whether parents had their children before or after their SCI.

\section{Fertility aids}

To explore reproductive techniques used by participants, a questionnaire was used. Response options for the method of ejaculation were normal, PVS, EE, or microsurgery. Response options for the fertilization method were home insemination, IUI, IVF, ICSI, or another individual method such as sperm donation or adoption. Men who reported using donor sperm or who had adopted their children were excluded from analyses regarding fertility aids.

\section{Factors associated with parenthood after SCI}

\section{Personal characteristics}

Information about current age, age at injury, menstruation in women (normal or changed after SCI), and current 
partnership was obtained using a questionnaire. Answer categories for partnership were 1) with partner (married or unmarried) and 2) without partner (single, divorced, or widow[er]).

\section{Disease characteristics}

Information was obtained about the cause of injury (traumatic or other) using a questionnaire and about the level and completeness of SCI using a neurological examination of the SCI according to the ISNCSCI classification and oral interview [21]. Participants were assessed for methods of micturition and defecation over the preceding 3 months using predefined response options. Micturition method could be answered as 1) normal or 2) abnormal: suprapubic tapping, intermittent catheterization, indwelling catheter, suprapubic catheter, urostomy, or neurostimulator for bladder control. Defecation method could be answered as (1) normal or (2) abnormal: abdominal massage or forward leaning, digital stimulation, contact irritants, manual evacuation, stool softeners, bowel irrigation system, colostomy, or sacral anterior root stimulation.

\section{Statistical analysis}

To interpret parenthood prevalence in SCI, we calculated 95\% confidence intervals (95\% CI) and compared prevalence rates in SCI with rates in the general Dutch population of the same median age. Parenthood prevalence rates of the Dutch population were obtained from the Dutch Central Agency for Statistics (years 2013 and 2014, $N=$ 130798 men and $N=130574$ women).

To explore the factors associated with parenthood after SCI, the study sample was divided into the following two groups: (1) persons who had become parents after SCI and (2) persons who had not become parents after SCI. Persons who had children before their SCI but not afterward were included in the latter group. Men and women were separately assessed using the Mann-Whitney $U$ test to explore whether age and age at injury differed between groups for those with and without children after SCI (not all data were normally distributed). The Pearson $X^{2}$ test was used to compare cause, level and completeness of SCI, bladder and bowel dysfunction, and civil status between groups with and without children after SCI.

To explore factors associated with parenthood, a logistic regression model was constructed for men and women separately. As we had no prespecified prediction hypothesis, we used a stepwise backward selection procedure to obtain the most parsimonious model for men and women separately. First, all variables (lesion level and completeness, cause of injury, bladder and bowel dysfunction, partnership, age at injury, and menstruation for women) were included in the regression model. Next, in a stepwise backward selection procedure, any variable in the multivariable model that was not significant at a $P$ value less than .05 was manually removed. Odds ratios were used to quantify associations between variables and parenthood. Descriptive statistics were used to quantify fertility aids used by men who fathered children after SCI. All statistical analyses were performed using SPSS version 19.0. Significance was assumed for $P$ values $<0.05$.

\section{Results}

A total of 282 persons participated in the national ALLRISC study. The data from 255 persons (90\%) were used in the current study. We excluded 27 persons for whom parenthood information was unavailable.

In Table 1 information on patient characteristics and parenthood is presented. Parenthood prevalence in men with SCI was $50 \%$ (95\% CI: 43-57\%), and in women with SCI this was $45 \%$ (95\% CI: $33-57 \%)$. These rates were significantly lower $(P<0.05)$ than in the Dutch general population, $74 \%$ (95\% CI: $74-74 \%$ ) in men and $81 \%$ (95\% CI: $81-81 \%)$ in women. Of parents with SCI, two-thirds had children after SCI (66\% of men and $72 \%$ of women). Of those who did not have children after SCI, $25 \%$ of men and $18 \%$ of women were already parents.

Table 2 (men) and Table 3 (women) show characteristics of the groups with and without children after SCI. Men who fathered children after SCI were less likely to have complete lesions $(P=.02)$, more likely to have normal defecation ( $P$ $=.01)$, and more likely to have a partner $(P<.001)$ compared to men who did not have children after SCI (Table 2). Women who had children after SCI were less likely to have traumatic SCI and more likely to have a partner $(P=.04)$ compared to women who did not have children after SCI (Table 3).

Logistic regression analysis showed that having a partner was associated with having children after SCI for both men $(\mathrm{OR}=14.5, P<.001)$ and women $(\mathrm{OR}=3.7, P=.05$; Table 4). Furthermore, normal micturition was associated with having children after $\mathrm{SCI}$ for men $(\mathrm{OR}=4.9, P=.02)$. Incomplete lesion $(\mathrm{OR}=5.4, P=.03)$ and lower lesion level $(\mathrm{OR}=7.3, P=.02)$ were associated with having children after SCI for women.

Table 5 presents the reproductive techniques used by men who fathered children after SCI $(n=63)$. Because six of those participants used donor sperm and one adopted a child, the analysis included 56 men. Of those 56 men, $41 \%$ used no ejaculation aid. The most frequently used methods for ejaculation were EE (29\%) and PVS (23\%), whereas the most frequently used method of fertilization was 
Table 1 Patient characteristics and parenthood in wheelchair-dependent persons with long-term spinal cord injury

\begin{tabular}{llll}
\hline & Total $N=255$ & Men $N=190(75 \%)$ & Women $N=65(25 \%)$ \\
\hline Patient characteristics & & & $48(42-55)$ \\
Age, (Q1-Q3), years & $48(42-55)$ & $23(20-28)$ & $23(21-26)$ \\
Age at injury, (Q1-Q3), y & $23(21-28)$ & 93 & 85 \\
Cause, \% traumatic & 91 & 44 & 30 \\
Level, \% tetraplegia & 41 & 68 & 69 \\
Completeness, \% complete & 69 & 6 & 14 \\
Micturition, \% normal & 8 & 12 & 14 \\
Defecation, \% normal & 13 & & \\
Partnership, \% & & 66 & 59 \\
$\quad$ With partner & 64 & 34 & 41 \\
$\quad$ Without partner & 37 & & $29(45)$ \\
Parenthood & $124(49)$ & $95(50)$ & $21(72)$ \\
Having children, $N(\%)$ & $84(68)$ & $63(66)$ & \\
Children born after SCI, $N$ (\% of parents) & & \\
\hline
\end{tabular}

Q1-Q3 interquartile 1-3, SCI spinal cord injury

Table 2 Characteristics of men with and without children after spinal cord injury $(N=190)$

Table 3 Characteristics of women with and without children after spinal cord injury $(N=65)$

\begin{tabular}{lllll}
\hline & $\begin{array}{l}\text { Children after SCI }(N= \\
\text { 63) }\end{array}$ & $\begin{array}{l}\text { No children after SCI }(N= \\
127)\end{array}$ & Test statistic & $P$ value \\
\hline Age, (Q1-Q3), years & $48(43-57)$ & $48(42-54)$ & -1.08 & 0.28 \\
Age at injury, (Q1-Q3), y & $21(20-28)$ & $23(22-28)$ & -1.28 & 0.20 \\
Cause, \% traumatic & 89 & 95 & 2.69 & 0.10 \\
Level, \% tetraplegia & 46 & 43 & 0.13 & 0.72 \\
Completeness, \% complete & 57 & 74 & 5.55 & 0.02 \\
Micturition, \% normal & 11 & 4 & 3.66 & 0.06 \\
Defecation, \% normal & 21 & 8 & 6.45 & 0.01 \\
Partnership & \% & & 32.51 & 0.001 \\
$\quad$ With partner & 93 & 52 & & \\
Without partner & 7 & 48 & & \\
\hline
\end{tabular}

$Q 1-Q 3$ interquartile 1-3, SCI spinal cord injury

${ }^{a} X^{2}$ based on the following two categories: (1) With partner (married/partner) and (2) without partner (divorced/widowed/single)

\begin{tabular}{lllll}
\hline & $\begin{array}{l}\text { Children after SCI }(N= \\
21)\end{array}$ & $\begin{array}{l}\text { No children after SCI }(N= \\
44)\end{array}$ & Test statistic & $P$ value \\
\hline Age, (Q1-Q3), years & $44(40-60)$ & $49(44-55)$ & -0.88 & 0.38 \\
Age at injury, (Q1-Q3), y & $23(20-24)$ & $25(21-27)$ & -1.81 & 0.07 \\
Cause, \% traumatic & 71 & 91 & 4.14 & 0.04 \\
Level, \% tetraplegia & 14 & 37 & 3.55 & 0.06 \\
Completeness, \% complete & 62 & 74 & 0.38 & 0.78 \\
Micturition, \% normal & 14 & 14 & 0.01 & 0.94 \\
Defecation, \% normal & 24 & 9 & 2.58 & 0.11 \\
Menstruation, \% normal & 57 & 57 & 0.00 & 0.98 \\
Partnership ${ }^{\text {a }}$ \%) & & & 4.01 & 0.04 \\
With partner & 76 & 50 & & \\
Without partner & 24 & 50 & & \\
\hline
\end{tabular}

Q1-Q3 interquartile 1-3, SCI spinal cord injury

${ }^{a} X^{2}$ based on the following two categories: (1) With partner (married/partner) and (2) without partner (divorced/widowed/single) 
Table 4 Logistic regression analyses for associated factors and parenthood in men and women with spinal cord injury

\begin{tabular}{llll}
\hline & OR & $\begin{array}{l}\text { 95\% Confidence interval } \\
\text { Lower-upper }\end{array}$ & $P$ value \\
\hline Men & & & \\
Partnership, partner & 14.5 & $5-43$ & 0.001 \\
Micturition, normal & 4.9 & $1-190$ & 0.02 \\
Women & & & 0.05 \\
Partnership, partner & 3.7 & $1-13$ & 0.03 \\
$\begin{array}{l}\text { Completeness, } \\
\text { incomplete }\end{array}$ & 5.4 & $1-25$ & 0.02 \\
Level, paraplegia & 7.3 & $1-40$ & \\
\hline
\end{tabular}

OR odds ratio

Table 5 Reproductive techniques used in men with children after spinal cord injury $(N=56)$

\begin{tabular}{ll}
\hline Reproductive technique & Number $(\%)$ \\
\hline Method of ejaculation & $23(41)$ \\
None used & $13(23)$ \\
Penile vibration stimulation & $16(29)$ \\
Electroejaculation & $2(4)$ \\
Microsurgery & $2(4)$ \\
n.a. & \\
Method of fertilization & $10(18)$ \\
Home insemination & $6(11)$ \\
IUI & $5(9)$ \\
IVF & $13(23)$ \\
ICSI & $10(18)$ \\
Other & $12(21)$ \\
n.a. ${ }^{a}$ & \\
\hline n.a. not assessed, IUI intrauterine insemination, IVF in vitro fertiliza- \\
tion, ICSI intracytoplasmatic sperm injection \\
a n.a. denotes the participants who did not complete the questionnaire \\
about use of ejaculation or fertilization aid
\end{tabular}

ICSI (23\%). Ten men used other methods, i.e., sexual intercourse $(n=9)$ or by using a cervical cup $(n=1)$.

\section{Discussion}

In this study, we investigated parenthood prevalence in a large group of wheelchair-dependent men and women with long-term SCI sustained during their reproductive years. Our sample was representative for the Dutch situation with regard to gender, level, and completeness of lesion [22]. We included a relatively high proportion of patients with a traumatic cause of SCI, but this is probably due to our focus on people who sustained their injury in their reproductive years. We found a parenthood prevalence of $50 \%$ in men and of $45 \%$ in women, which was low when compared to the Dutch general population $(74 \%$ in men and $81 \%$ in women). However, to reframe this finding, half of the participants with SCI do become parents, with two-thirds of those parents having children after SCI. For both men and women, parenthood was associated with having a partner. Additionally, normal micturition was associated with parenthood in men, and incomplete lesion and lower lesion level with parenthood in women. We further found that fathers most frequently used electroejaculation to aid fertility and ICSI with their partners for fertilization. Surprisingly, many fathers $(41 \%)$ used no reproductive aid.

Given the known fertility problems for men with SCI $[12,23,24]$, the low prevalence of parenthood fits with our hypothesis. However, we did not expect that the prevalence of parenthood in women would also be as low as men because fertility after SCI returns to levels comparable to those of able-bodied women following a short period of amenorrhea [4, 5]. We showed that, in addition to partnership, lesion severity was associated with parenthood for women with SCI. One possible explanation for this finding is that women with more severe SCI have more concerns about their ability to care for a child. Pebdani et al. previously showed that women, more than men, consider their SCI in decisions about family planning [17]. As expected, having a partner was associated with parenthood in both men and women. Lezonni et al. [25], who examined pregnancy prevalence among women with SCI, also found a strong relationship with partnership. In the Dutch general population, only $17 \%$ of persons aged 30-65 years are single [26], whereas $37 \%$ of patients with SCI in our study did not have a partner.

For men with SCI, we have found an association between micturition and parenthood. Men with normal micturition are more likely to have normal fertility; thus, they have a greater chance of becoming fathers [27, 28]. However, these findings should be interpreted with caution because micturition responses pertained only to the preceding 3 months. Micturition or bladder control may have differed at the time their children were conceived.

With respect to the use of fertility aids by men with SCI who fathered children, we expected that most of them had used assisted reproductive technology for ejaculation or fertilization. Surprisingly, $41 \%$ of those men used no assistance for ejaculation or fertilization. Generally, male SCI fertility rates are less than $10 \%$ among those who do not use assistive technology. The striking difference between our findings and those published previously [4, 29, 30] may be explained by different study populations. Most published fertility rates are for men with SCI who require assisted reproductive techniques for fertilization or ejaculation (thus the presence of fertility problems is implied), 
whereas our study sample includes a more diverse population of men affected by SCI.

We feel that our findings are of clinical importance. The information obtained through this study is important to create awareness, both in healthcare professionals and people with SCI, on parenthood prevalence and fertility aids. Furthermore, the study can be used to facilitate counseling for people with SCI who wish to have children, with focus on the opportunities of becoming a parent.

However, some limitations should be considered. First, able-bodied parent data obtained from the Dutch Central Agency for Statistics (years 2013-2014) were chosen at ages similar to the median age of people with SCI included in our study. Because our sample included patients younger than this median age, parenthood in SCI may be underestimated in the current study. However, the able-bodied parenthood prevalence rates may be underestimated as well, since the Dutch general population data comprise information for both able-bodied and disabled persons. Second, the national ALLRISC study, of which this study was a part, was designed to evaluate long-term functioning in patients with SCI. Fertility after SCI was not the main focus of the ALLRISC study. Thus, the set of associated factors that we studied was not comprehensive and was exploratory in nature. Furthermore, the ALLRISC study had a crosssectional design. Third, given the small sample size of women who mothered children after SCI $(n=21)$, factors associated with parenthood in this subsample should be interpreted with caution. Given the limitations, the current study should be considered as an initial step in the research on prevalence of parenthood in persons with SCI. Future research should include longitudinal designs with a comprehensive set of factors that may be associated with parenthood prevalence.

In conclusion, our study shows that the prevalence of parenthood among men and women with SCI is low. However, approximately half of the persons with SCI do become parents, with most doing so following SCI.

Acknowledgements This project is part of the Dutch ALLRISC research program and is financially supported by ZonMw Rehabilitation program and Fonds NutsOhra, grant no. 89000006.

\section{Compliance with ethical standards}

Conflict of interest The authors declare that they have no conflict of interest.

\section{References}

1. Asbeck WFv, Nes IJWv. Handboek dwarslaesierevalidatie. 3rd ed. Houten: Bohn Stafleu van Loghum; 2007.
2. Brackett NL, Lynne CM, Ibrahim E, Ohl DA, Sonksen J. Treatment of infertility in men with spinal cord injury. Nat Rev Urol. 2010;7:162-72.

3. National Spinal Cord Injury Statistical Center. Facts and figures at a glance. Birmingham: University of Alabama; 2013.

4. DeForge D, Blackmer J, Garritty C, Yazdi F, Cronin V, Barrowman N, et al. Fertility following spinal cord injury: A systematic review. Spinal Cord. 2005;43:693-703.

5. Sipski M. The impact of spinal cord injury on female sexuality, menstruation and pregnancy: a review of the literature. J Am Paraplegia Soc. 1991;14:122-6.

6. Brackett NL, Nash MS, Lynne CM. Male fertility following spinal cord injury: facts and fiction. Phys Ther. 1996;76:1221-31.

7. Dimitriadis F, Karakitsios K, Tsounapi P, Tsambalas S, Loutradis D, Kanakas N, et al. Erectile function and male reproduction in men with spinal cord injury: a review. Andrologia. 2010;42:139-65.

8. Raviv G, Madgar I, Elizur S, Zeilig G, Levron J. Testicular sperm retrieval and intra cytoplasmic sperm injection provide favorable outcome in spinal cord injury patients, failing conservative reproductive treatment. Spinal Cord. 2013;51:642-4.

9. Nehra A, Werner MA, Bastuba M, Title C, Oates RD. Vibratory stimulation and rectal probe electroejaculation as therapy for patients with spinal cord injury: Semen parameters and pregnancy rates. J Urol. 1996;155:554-9.

10. Dahlberg A, Ruutu M, Hovatta O. Pregnancy results from a vibrator application, electroejaculation, and a vas aspiration programme in spinal-cord injured men. Hum Reprod. 1995;10:2305-7.

11. Shieh JY, Chen SU, Wang YH, Chang HC, Ho HN, Yang YS. A protocol of electroejaculation and systematic assisted reproductive technology achieved high efficiency and efficacy for pregnancy for anejaculatory men with spinal cord injury. Arch Phys Med Rehabil. 2003;84:535-40.

12. Brackett NL, Ibrahim E, Iremashvili V, Aballa TC, Lynne CM. Treatment for ejaculatory dysfunction in men with spinal cord injury: An 18-year single center experience. J Urol. 2010;183:2304-8.

13. Hultling C, Rosenlund B, Levi R, Fridstrom M, Sjoblom P, Hillensjo T. Assisted ejaculation and in-vitro fertilization in the treatment of infertile spinal cord-injured men: The role of intracytoplasmic sperm injection. Hum Reprod. 1997;12:499-502.

14. Merghati Khoi E, Latifi S, Rahdari F, Shakeri H, Arman F, Koushki D, et al. The Effect of injury-related characteristics on changes in marital status after spinal cord injury. Iran J Public Health. 2015;44:1395-402.

15. Jorgensen S, Iwarsson S, Norin L, Lexell J. The Swedish aging with spinal cord injury study (SASCIS): Methodology and initial results. PM R. 2016;8:667-77.

16. Cross LL, Meythaler JM, Tuel SM, Cross AL. Pregnancy, labor and delivery post spinal cord injury. Paraplegia. 1992;30:890-902.

17. Pebdani RL, Johnson KL, Amtmann D. Personal experiences of pregnancy and fertility in individuals with spinal cord injury. Sex Disabil. 2014;32:65-74.

18. Nikas V, Fleischman P, Burton P. Sexual and marital adjustment of World War II spinal cord injured veterans. Paraplegia. 1990;28:164-71.

19. Center TNSCIS. Annual statistical report for the spinal cord injury model systems-complete public version. Birmingham: University of Alabama; 2012.

20. Adriaansen J, WFv Asbeck, Lvd Woude, Groot Sd. Secondary health conditions in persons with a spinal cord injury for at least 10 years: design of a comprehensive long-term corss-sectional study. Disabil Rehabil. 2013;35:1104-10. 
21. Kirshblum SC, Burns SP, Biering-Sorensen F, Donovan W, Graves DE, Jha A, et al. International standards for neurological classification of spinal cord injury (revised 2011). J Spinal Cord Med. 2011;34:535-46.

22. de Groot S, Dallmeijer AJ, Post MW, van Asbeck FW, Nene AV, Angenot EL, et al. Demographics of the Dutch multicenter prospective cohort study 'Restoration of mobility in spinal cord injury rehabilitation'. Spinal Cord. 2006;44:668-75.

23. Monga M, Bernie J, Rajesekaran M. Male infertility and erectile dysfunction in spinal cord injury: a review. Arch Phys Med Rehabil. 1999;80:1331-9.

24. Brackett NL, Lynne CM, Ibrahim E, Ohl DA, Sonksen J. Treatment of infertility in men with spinal cord injury. Nat Rev Urol. 2010;7:162-72.

25. Lezzoni LI, Chen Y, McLain AB. Current pregnancy among women with spinal cord injury: findings from the US national spinal cord injury database. Spinal Cord. 2015;53:821-6.
26. CBS. Central agency for statistics 2015. Statistics Netherlands; 2015.

27. Dimitriadis F, Karakitsios K, Tsounapi P, Tsambala S, Loutradis D, Kanakas N, et al. Erectile function and male reproduction in men with spinal cord injury: a review. Andrologia. 2009;42:139-65.

28. Rutkowski SB, Middleton JW, Truman G, Hagen DL, Ryan JP. The influence of bladder management on fertility in spinal cord injured males. Paraplegia. 1995;33:263-6.

29. Taylor Z, Molloy D, Hill V, Harrison K. Contribution of the assisted reproductive technologies to fertility in males suffering spinal cord injury. Aust N Z J Obstet Gynaecol. 1999;39:84-7.

30. Rutkowski SB, Geraghty TJ, Hagen DL, Bowers DM, Craven M, Middleton JW. A comprehensive approach to the management of male infertility following spinal cord injury. Spinal Cord. 1999;37:508-14. 